\title{
COGNITIONS AND PERCEPTIONS OF HEALTH AND EXERCISE
}

\author{
S. J. H. BIDDLE* and B. ASHFORD** \\ *University of Exeter and * North Staffordshire Polytechnic, Stoke-on-Trent
}

\section{ABSTRACT}

The impact of exercise on health is potentially significant yet relatively few people exercise. The paper reports two exploratory, community-based, cross-sectional retrospective surveys which investigated the cognitions of aerobic exercisers and non-exercisers. Specifically, an analysis was undertaken of differences between exercisers and non-exercisers, between males and females, and between those under 40 and those over 40 years of age on health beliefs, exercise cognitions, knowledge and attributions. Results from Study $1(N=433)$ showed clear differences between exercise, age and gender groups on health beliefs, knowledge and attributions with exercisers having a more positive cognitive profile. Non-exercisers, contrary to the Health Belief Model, were characterised by perceptions of vulnerability to general and cardiac ill-health. Study $2(N=468)$ supported these results and also showed that older people had more negative beliefs and worries about exercise than younger people. Discussion of the results centres on the need for further research on the causality and prediction of exercise and other health-related behaviours from cognitive factors.

Key words: Attributions, Cognitions, Exercise psychology, Health beliefs

It is now recognised fully that the contribution of physical activity to health and well-being is potentially significant. In 1980, the United States Government Department of Health and Human Services (DHHS) published its national health objectives for the 1990s, eleven of which specifically referred to physical fitness and exercise (DHHS, 1980). Considerable progress has been made in meeting some of these objectives by some sections of the American population (Powell et al, 1986). Unfortunately, there is little data concerning the British population.

Whatever strategy is adopted to change an individual's health through regular participation in exercise, the critical question now is not whether to exercise but how best to promote exercise behaviour. This requires further research into the psychology of health-related exercise.

Although sport psychologists have only recently investigated health-related issues there has been a concerted research effort in health psychology for many years. One such effort has been concerned with individual beliefs and cognitions concerned health and whether such factors are predictive of health behaviours.

During the 1950s, a group of American social psychologists attempted to integrate the diverse findings of research on health behaviour. Such diversity led these researchers to develop The Health Belief Model [(HBM) Becker et al, 1977; Janz and Becker, 1984].

The HBM hypothesises that people will not seek preventive health behaviours unless they:

1. Possess at least minimal levels of health motivation and knowledge;

2. View themselves as potentially vulnerable;

3. View the condition as threatening;

4. Are convinced of the efficacy of the treatment;

5. See few difficulties in undertaking the action.

These factors can be modified by socio-economic and demographic factors, as well as 'cues to action', such as media campaigns or the illness of a friend. Such variables

Address for correspondence:

Stuart Biddle, PhD

School of Education

University of Exeter

Exeter

EX12LU may provide a useful framework for the study of behaviours associated with health-related exercise.

The HBM was devised to account primarily for avoidance behaviours. This can be seen in the assumptions that health action will be somewhat dependent on perceptions of vulnerability and susceptibility to ill-health. Nevertheless, it has been tested in other health behaviour contexts, such as participation in physical exercise.

Lindsay-Reid and Osborn (1980), in a study of Canadian firemen, found only weak support for a relationship between the perceived benefits of exercise and exercise adoption. However, contrary to the HBM they also found that exercise was negatively associated with perceptions of susceptibility to coronary heart disease (CHD) and general illnesses.

Slenker and co-workers, in a study of joggers and nonexercisers, found that 'barriers to action' (e.g. time, weather, responsibilities, etc.), was a strong predictor variable in jogging behaviour (Slenker et al, 1984). This was supportive of earlier work by Riddle (1980) who found that non-exercisers thought that jogging would require too much time, would make them too tired, and would take too much discipline. Similarly, Godin and co-workers found that non-exercisers who intended to exercise perceived exercise as physically demanding and time-consuming (Godin et al, 1986).

Belief in the benefits of physical exercise, as well as confidence in the ability to control health outcomes, were predictive of participation in a North American employee fitness programme (Morgan et al, 1984). However, others have found only weak support for the HBM in predicting exercise behaviours of obese and non-obese adolescents (O'Connell et al, 1985). Similarly, exploratory testing of a modified version of the HBM for exercise (Noland and Feldman, 1984, 1985) found that only locus of control and physical activity attitude variables predicted exercise involvement. The addition of locus of control measures is potentially significant as King (1982) found that attenders and non-attenders of a blood pressure screening clinic could be discriminated between on health belief and attributional (control) variables. Other research has also supported the use of variables which assess perceptions of causality and control in illness, disease and health-related behaviours (e.g. Bar-On and Cristal, 1987).

The purpose of this paper, therefore, is to explore any 
differences between exercisers and non-exercisers in beliefs and attributions concerning health and exercise. Two cross-sectional retrospective surveys are reported.

\section{STUDY 1}

Given the interest in and importance of $\mathrm{CHD}$ and the potentially significant role of exercise in reducing CHD risk (Powell et al, 1987), Study 1 investigated beliefs, knowledge and attributions associated with cardiovascular, as well as general, health. Specifically, the study had the following aims:

1. To ascertain the nature of differences, if any, between aerobic exercisers and non-exercisers in health beliefs, knowledge and attributions concerning cardiovascular health;

2. To ascertain the nature of differences, if any, between men and women on the same variables;

3. To ascertain the nature of differences, if any, between those under 40 years of age and those over 40 years, again on the same variables.

\section{Method}

The study was based on a retrospective design and a streetbased questionnaire-interview of approximately 5 minutes duration and was conducted in seven urban locations in the Stoke-on-Trent and Newcastle-under-Lyme conurbation in Staffordshire, England on one Saturday in March. The sample comprised 433 people (234 men, 199 women). 263 were under the age of 40,170 were over 40 years of age. All subjects were over 16 years of age. Accidental sampling was used but a reasonably representative cross-section of age, gender and racial groups was attempted.

\section{Questionnaire}

In addition to personal descriptive data, the questionnaire assessed beliefs about general and cardiovascular health, intention to attend a hypothetical cardiac screening clinic, health attributions, and knowledge of health (14 true/false questions primarily on CHD risk and yielding one knowledge score). Each subject was also asked how frequently they took part in aerobic-type physical exercise. Subjects were asked for their perceptions of: general health vulnerability, health saliency, health motivation, heart concern, past health behaviour, perceived barriers to attending the clinic, time since last cardiac check-up, intention to attend a screening session, perceived vulnerability to $\mathrm{CHD}$, perceived severity of CHD should it occur, time since last cardiac check-up, intention to attend a screening session, perceived vulnerability to CHD, perceived severity of CHD should it occur, time since personal CHD symptoms suffered and time since family or friends suffered CHD symptoms. Attribution questions were asked about: locus of causality of CHD (internal or external influence), the extent to which causes of cardiovascular health are perceived as stable or unstable, and the extent to which cardiovascular health is perceived to be controllable or uncontrollable.

All questions were inspected for clarity and appropriateness by two researchers and a large number of students, who were briefed in preparation for the street interviews.

Data were analysed by a $2 \times 2 \times 2$ (gender $\times$ age $\times$ exercise frequency) MANOVA. In order to distinguish clearly exercisers from non-exercisers, the exercise frequency variable was recoded prior to the MANOVA. Two groups were thus created: 'exercisers' were classified as those who participated at least twice a week $(N=164)$ and 'non- exercisers' were those who participated 'occasionally' or 'never' ( $N=177)$. The 92 subjects who were omitted from all analyses exercised only once per week or once per month.

\section{Results}

The $2 \times 2 \times 2$ MANOVA analysed all health belief and attribution variables, as well as health knowledge. There were significant multivariate main effects for all three independent variables, as well as significant multivariate age $\times$ exercise and gender $\times$ exercise interactions. Descriptive data are shown in Table 1.

\section{Aim 1: Exercisers versus non-exercisers}

The main effect for exercise showed that exercisers and non-exercisers were significantly different in their health cognitions $(P<0.0001)$. Exercisers were higher in knowledge, health motivation, and perceptions of control, as well as having done more in the past to maintain their cardiovascular health. Non-exercisers, on the other hand, had greater perceptions of vulnerability to general and cardiac ill-health and perceived greater barriers to attending a hypothetical cardiac screening clinic.

\section{Aim 2: Gender differences}

There was a significant main effect for gender $(P<0.001)$. This showed that women were significantly higher in perceptions of general health vulnerability and health concern, whereas men were higher in health motivation, perceptions of heart vulnerability and were more likely to have done something about cardiovascular health in the past.

\section{Aim 3: Age differences}

The significant main effect for age $(P<0.0001)$ revealed differences on 10 variables. Subjects under 40 years of age were higher on knowledge, health motivation, and perceived benefits of action. Those over 40 years had higher perceptions of general health and heart vulnerability, heart concern, and health saliency, and were more likely to have had a recent cardiovascular check-up as well being more likely to have suffered recent cardiovascular health problems either themselves or by their close family or friends.

\section{Interaction effects}

The gender $\times$ exercise interaction $(P<0.05)$ revealed three significant univariate interactions. These showed: (i) the greatest barriers to attendance at the cardiac screening clinic were perceived by non-exercising women; (ii) perceived vulnerability to heart problems was greatest in non-exercising males; (iii) more recent cardiovascular health problems of family and friends were reported by exercising females and non-exercising males than by other groups.

The multivariate age $\times$ exercise interaction $(P<0.02)$ revealed interactions on two variables. These showed: (i) the greatest barriers to clinic attendance were perceived by exercisers over 40 years of age; (ii) less control was perceived over their cardiovascular health by nonexercisers over 40 years of age than by other groups.

\section{DISCUSSION}

This exploratory study has shown that differences are evident between aerobic exercisers and non-exercisers on a 
TABLE I

Means and standard deviations of dependent variables used in Study 1

\begin{tabular}{|c|c|c|c|c|c|c|c|c|c|c|c|c|}
\hline \multirow{3}{*}{ VARIABLE } & \multicolumn{4}{|c|}{ EXERCISE } & \multicolumn{4}{|c|}{ GENDER } & \multicolumn{4}{|c|}{ AGE } \\
\hline & \multicolumn{2}{|c|}{ Exercisers ( $N=164)$} & \multicolumn{2}{|c|}{ Non-exercisers $(N=177)$} & \multicolumn{2}{|c|}{ Male $(N=190)$} & \multicolumn{2}{|c|}{ Female $(N=151)$} & \multicolumn{2}{|c|}{ Under $40(N=192)$} & \multicolumn{2}{|c|}{ Over $40(N=149)$} \\
\hline & M & SD & M & SD & $\mathbf{M}$ & SD & $\mathbf{M}$ & SD & $M$ & SD & $\mathbf{M}$ & SD \\
\hline Knowledge $(1,3)$ & 9.25 & 2.08 & 8.31 & 2.12 & 8.75 & 2.14 & 8.76 & 2.17 & 9.08 & 2.09 & 8.34 & 2.16 \\
\hline $\begin{array}{l}\text { General health } \\
\text { vulnerability }(1,2,3)\end{array}$ & 1.96 & 1.18 & 2.71 & 1.08 & 2.17 & 1.19 & 2.58 & 1.16 & 2.06 & 1.05 & 2.74 & 1.25 \\
\hline Health saliency (3) & 2.44 & 1.16 & 2.40 & 1.08 & 2.41 & 1.17 & 2.43 & 1.04 & 2.22 & 1.08 & 2.66 & 1.12 \\
\hline Health motivation $(1,2,3)$ & 3.73 & 1.04 & 2.38 & 1.09 & 3.26 & 1.29 & 2.74 & 1.17 & 3.32 & 1.26 & 2.66 & 1.17 \\
\hline Heart concern $(2,3)$ & 2.23 & 1.25 & 2.30 & 1.35 & 2.11 & 1.26 & 2.46 & 1.33 & 2.07 & 1.23 & 2.52 & 1.34 \\
\hline $\begin{array}{l}\text { Past health behaviour } \\
(1,2)\end{array}$ & 3.43 & 1.26 & 2.20 & 1.17 & 3.05 & 1.37 & 2.47 & 1.28 & 3.00 & 1.43 & 2.52 & 1.21 \\
\hline Barriers (1) & 3.44 & 1.57 & 3.15 & 1.47 & 3.37 & 1.54 & 3.19 & 1.51 & 3.19 & 1.56 & 3.41 & 1.48 \\
\hline Behavioural intention & 3.67 & 1.32 & 3.12 & 1.38 & 3.41 & 1.40 & 3.35 & 1.35 & 3.38 & 1.38 & 3.39 & 1.38 \\
\hline Previous action (3) & 3.09 & 1.16 & 3.08 & 1.09 & 3.14 & 1.12 & 3.02 & 1.13 & 3.26 & 1.10 & 2.86 & 1.12 \\
\hline $\begin{array}{l}\text { Heart vulnerability } \\
(1,2,3)\end{array}$ & 2.58 & 0.99 & 2.94 & 0.97 & 2.87 & 1.09 & 2.64 & 0.85 & 2.55 & 0.98 & 3.05 & 0.96 \\
\hline Heart severity & 2.86 & 0.91 & 3.03 & 0.90 & 2.99 & 0.99 & 2.91 & 0.80 & 2.83 & 0.89 & 3.11 & 0.91 \\
\hline Benefit (3) & 4.07 & 1.11 & 3.76 & 1.24 & 3.94 & 1.21 & 3.87 & 1.16 & 4.11 & 1.07 & 3.65 & 1.27 \\
\hline Personal symptoms (3) & 3.81 & 0.66 & 3.67 & 0.82 & 3.70 & 0.79 & 3.79 & 0.69 & 3.90 & 0.50 & 3.52 & 0.95 \\
\hline Family symptoms (3) & 2.93 & 1.14 & 2.77 & 1.20 & 2.80 & 1.17 & 2.91 & 1.18 & 3.05 & 1.14 & 2.58 & 1.16 \\
\hline Locus attribution & 3.10 & 1.22 & 2.91 & 1.25 & 2.99 & 1.27 & 3.01 & 1.19 & 3.04 & 1.27 & 2.95 & 1.19 \\
\hline Stability attribution & 3.83 & 1.13 & 3.79 & 1.30 & 3.85 & 1.23 & 3.76 & 1.21 & 3.72 & 1.21 & 3.93 & 1.22 \\
\hline $\begin{array}{l}\text { Controllability } \\
\text { attribution (1) }\end{array}$ & 3.75 & 1.01 & 3.38 & 1.27 & 3.55 & 1.11 & 3.57 & 1.23 & 3.72 & 1.06 & 3.35 & 1.26 \\
\hline
\end{tabular}

Notes: (1) Difference between exercisers and non-exercisers $P<0.05$

(2) Difference between males and females $P<0.05$

(3) Difference between age groups $P<0.05$

(4) All variables assessed on 5-point scales except knowledge (out of 14), and personal and family symptoms (4 point scales)

number of variables associated with cardiac and general health, knowledge and attributions. In particular, exercisers compared with non-exercisers were characterised by being more motivated and knowledgeable, having done something in the past to maintain cardiac health and naving higher perceptions of control over their own health. However, non-exercisers were actually above the scale midpoints for knowledge and perceptions of control. Nonexercisers were also characterised, relative to exercisers, by higher perceptions of vulnerability to both general ill-health and cardiac disease and perceived greater barriers to attendance at a hypothetical cardiac screening clinic.

These results show that exercisers have a more positive psychological profile of health beliefs than non-exercisers. However, it is not known to what extent these links are causal, nor is it known whether such a cognitive profile is the result of, or antecedent to, exercise participation. Nevertheless, the differences are noteworthy and justify further research.

The results for non-exercisers show that perceptions of vulnerability are associated with a lack of health action, at least as far as aerobic exercise is concerned. Although the scores for these variables, as shown in Table I, are not particularly high in an absolute sense, the relative difference between exercisers and non-exercisers is noteworthy.

The gender differences appear to reflect the greater prevalence of CHD among males since it was they who had greater perceptions of vulnerability to CHD, although women were higher on general health vulnerability and, surprisingly, concern about CHD. However, men were more likely to have done something in the past to improve their cardiac health than women. Although the incidence of CHD is greatest in men, $23 \%$ of deaths in women are due to CHD (British Cardiac Society, 1986). CHD in women has not been given as much publicity as for men and this could account for some of the different perceptions reported here.

The younger age group generally displayed a more positive cognitive profile than older subjects. In particular, they were more knowledgeable, motivated and perceived greater benefits in taking action for cardiac health. This could be due to higher educational attainment (the younger subjects in the sample had a higher educational level) and/ or changing societal attitudes. This requires further study. Although the older age group had a higher health saliency, they also felt greater vulnerability to both general and cardiac ill-health. This was probably associated with their perceptions (correctly in this case) of increasing CHD risk with increasing age. However, the age $\times$ exercise interaction on perceptions of health control suggested that older subjects who did not exercise felt less control over their own health. This could lead to a lack of health action such as exercise. The results for age differences, however, suggest that more work is required in educating older adults about appropriate forms of health-related exercise, preferably by having education programmes for young people which emphasise the importance of lifetime participation in exercise.

\section{STUDY 2}

Study 1 showed that health and exercise beliefs differed between exercisers and non-exercisers. However, much of the focus in Study 1 was on beliefs concerning cardiovascular health, and the behavioural intention variable referred to attendance at a hypothetical cardiac screening 
clinic. Study 2, therefore, was conducted to extend the investigation and to ask more specific questions about exercise beliefs, in addition to beliefs concerning general and cardiovascular health. The question concerning behavioural intention referred to exercise. Additionally, three attribution variables were included. In Study 1, measures of locus of causality (internal/external), stability (stable/unstable) and controllability (controllable/uncontrollable) were assessed. These are the three traditional dimensions used in achievement attribution research, including sport (see Weiner, 1986). However, King (1982) found that other attribution variables were more predictive of health behaviours. These were whether people used 'consensus' information (the degree to which they believe other people act in the same way), 'consistency' information (the degree to which an individual has behaved in a similar way in the past), and 'distinctiveness' information (the degree to which an individual acts in similar ways in different situations, such as eating 'healthy' foods as well as exercising; see Biddle, 1986). These three variables were added to the questionnaire.

Study 2, therefore, sought to extend Study 1 and had two main aims:

1. To ascertain the nature of differences, if any, between aerobic exercisers and non-exercisers in health and exercise beliefs, and health knowledge and attributions;

2. To ascertain the nature of gender and age differences, if any, on the same variables.

\section{METHOD}

The method adopted in Study 1 was replicated. Questionnaire-interviewing was carried out in Birmingham city centre (England) on one mid-week day in April. 468 subjects took part (238 men; 230 women) and all were over the age of 16 years.

\section{Questionnaire}

The variables used in the questionnaire/interview were as follows: health knowledge, general health vulnerability, health saliency, health motivation, heart concern, past exercise behaviour, perceived barriers to exercise, intention to exercise, perceived vulnerability to CHD, perceived severity of CHD should it occur, exercise benefits, exercise importance and exercise safety. The following attribution variables were assessed: locus of causality, stability, controllability (as in Study 1), and consensus, consistency, and distinctiveness information, as described above. The health knowledge score was derived from 6 true/false questions. Again, subjects were asked how frequently they took part in aerobic-type physical activity.

As in Study 1, in order to clearly distinguish 'exercisers' from 'non-exercisers', the exercise group was classified as those subjects who participated in aerobic-type exercise at least twice a week ( $N=232)$ and non-exercisers were classified as those-who participated 'occasionally' or 'never' $(N=172)$. Subjects were therefore omitted from analyses if they exercised only once a week or once per month. Unfortunately, the frequencies obtained in some cells in a $2 \times 2 \times 2$ (gender $\times$ age $\times$ exercise frequency) analysis were close to zero. Given the increasing interest in exercise for older people, and the changing age profile of the population, it was decided to keep age as an independent variable and to combine scores across gender groups. The data were therefore analysed using a $2 \times 2$ (age $\times$ exercise frequency) MANOVA with health and exercise beliefs, knowledge, and attributions as the dependent variables.

\section{RESULTS}

The $2 \times 2$ MANOVA revealed significant multivariate main effects for both age and exercise frequency, and also a significant age $\times$ exercise interaction. Data are shown in Table II.

TABLE II

Means and standard deviations of dependent variables used in Study 2

\begin{tabular}{|c|c|c|c|c|c|c|c|c|}
\hline \multirow[b]{3}{*}{ Variable } & \multicolumn{4}{|c|}{ EXERCISE } & \multicolumn{4}{|c|}{ AGE } \\
\hline & \multicolumn{2}{|c|}{$\begin{array}{l}\text { Exercisers } \\
(N=232)\end{array}$} & \multicolumn{2}{|c|}{$\begin{array}{c}\text { Non- } \\
\text { exercisers } \\
(N=172)\end{array}$} & \multicolumn{2}{|c|}{$\begin{array}{l}\text { Under } 40 \\
(N=302)\end{array}$} & \multicolumn{2}{|c|}{$\begin{array}{l}\text { Over } 40 \\
(N=102)\end{array}$} \\
\hline & $\mathbf{M}$ & SD & $\mathbf{M}$ & SD & $\mathbf{M}$ & SD & $\mathbf{M}$ & SD \\
\hline $\begin{array}{l}\text { Health } \\
\text { saliency }(1,2)\end{array}$ & 3.14 & 1.25 & 2.69 & 1.28 & 2.85 & 1.25 & 3.21 & 1.33 \\
\hline $\begin{array}{l}\text { Health } \\
\text { motivation (1) }\end{array}$ & 3.87 & 1.09 & 2.70 & 1.05 & 3.50 & 1.16 & 3.00 & 1.31 \\
\hline $\begin{array}{l}\text { Past } \\
\text { behaviour (1) }\end{array}$ & 4.01 & 1.13 & 1.88 & 1.10 & 3.24 & 1.52 & 2.69 & 1.46 \\
\hline $\begin{array}{l}\text { Exercise } \\
\text { intention (1) }\end{array}$ & 4.03 & 1.26 & 2.05 & 1.10 & 3.39 & 1.52 & 2.57 & 1.44 \\
\hline $\begin{array}{l}\text { Exercise } \\
\text { importance (1) }\end{array}$ & 4.34 & 1.05 & 2.83 & 1.38 & 3.76 & 1.40 & 3.49 & 1.44 \\
\hline $\begin{array}{l}\text { General health } \\
\text { vulnerability (1) }\end{array}$ & 2.07 & 1.26 & 2.50 & 1.19 & 2.16 & 1.26 & 2.53 & 1.16 \\
\hline Heart concern (2) & 2.43 & 1.41 & 2.21 & 1.34 & 2.23 & 1.37 & 2.65 & 1.38 \\
\hline $\begin{array}{l}\text { Heart } \\
\text { vulnerability (2) }\end{array}$ & 2.40 & 1.18 & 2.77 & 1.02 & 2.47 & 1.20 & 2.80 & 1.13 \\
\hline Heart severity (2) & 2.63 & 1.21 & 2.79 & 1.08 & 2.63 & 1.15 & 2.90 & 1.15 \\
\hline $\begin{array}{l}\text { Exercise } \\
\text { benefits (1) }\end{array}$ & 4.44 & 0.94 & 3.55 & 1.21 & 4.15 & 1.09 & 3.70 & 1.28 \\
\hline Exercise barriers & 2.83 & 1.57 & 3.27 & 1.54 & 2.91 & 1.55 & 3.32 & 1.59 \\
\hline Exercise safety (2) & 4.41 & 0.96 & 3.86 & 1.15 & 4.34 & 0.98 & 3.68 & 1.15 \\
\hline Knowledge (2) & 3.50 & 1.22 & 3.08 & 1.20 & 3.41 & 1.24 & 3.04 & 1.15 \\
\hline $\begin{array}{l}\text { Locus } \\
\text { attribution (1) }\end{array}$ & 4.04 & 1.21 & 3.29 & 1.37 & 3.78 & 1.34 & 3.54 & 1.31 \\
\hline $\begin{array}{l}\text { Stability } \\
\text { attribution }\end{array}$ & 2.70 & 1.53 & 2.74 & 1.49 & 2.83 & 1.54 & 2.36 & 1.39 \\
\hline $\begin{array}{l}\text { Controllability } \\
\text { attribution (1) }\end{array}$ & 4.10 & 1.11 & 3.66 & 1.29 & 3.93 & 1.19 & 3.86 & 1.25 \\
\hline $\begin{array}{l}\text { Consensus } \\
\text { information (1) }\end{array}$ & 2.91 & 1.35 & 2.69 & 1.40 & 2.83 & 1.37 & 2.78 & 1.36 \\
\hline $\begin{array}{l}\text { Consistency } \\
\text { information (1) }\end{array}$ & 4.16 & 1.13 & 1.66 & 0.89 & 3.23 & 1.59 & 2.69 & 1.64 \\
\hline $\begin{array}{l}\text { Distinctiveness } \\
\text { information }\end{array}$ & 3.20 & 1.29 & 3.31 & 1.30 & 3.27 & 1.25 & 3.20 & 1.42 \\
\hline
\end{tabular}

Notes: (1) Difference between exercisers and non-exercisers $P<0.05$ (2) Difference between age groups $P<0.05$

(3) All variables assessed on 5 point scales except knowledge, which was the number of correct answers out of six

\section{Aim 1: Exercisers versus non-exercisers}

Exercisers and non-exercisers were significantly different in their multivariate profiles $(P<0.0001)$. Univariate analyses showed that exercisers were higher in perceptions of exercise intention, importance and benefits, as well as having higher health motivation (as in Study 1) and health saliency. Additionally, they were shown to have higher beliefs in exercise control (thus replicating the results from Study 1), were more likely to have been active in the past, and were more likely to have modified other health habits. Non-exercisers had higher perceptions of general health vulnerability. This is also supportive of Study 1.

\section{Aim 2: Age differences}

The two age groups (under and over 40 years) were 
significantly different when their multivariate profiles were compared $(P<0.0001)$. Univariate analyses showed that the older group had higher perceptions of health saliency, heart concern, heart vulnerability and heart severity than the younger group. The younger group had better knowledge and felt exercise was safer. The results for knowledge, saliency, concern and vulnerability replicate those found in Study 1.

\section{Interaction effects}

The significant age $\times$ exercise frequency interaction $(P<$ 0.001 ) revealed that the intention to exercise was highest in exercisers under 40 years of age. Also, other health behaviours were less likely to be adopted by those over $\mathbf{4 0}$ years of age who were non-exercisers.

\section{DISCUSSION}

A clear difference between exercisers and non-exercisers was found which corroborates the results of Study 1. Again, non-exercisers were found to have higher perceptions of vulnerability to general ill-health. Exercisers, on the other hand, had a very positive profile of exercise beliefs, being higher in intention, importance and benefits. They also had stronger beliefs in controlling their own behaviour for exercise. The inclusion of these, and the additional attributional variables, was therefore vindicated. Exercisers were higher on 'consensus' beliefs. That is to say they were more likely to have adopted other health behaviours in addition to exercise. Although causality cannot be inferred, this suggests that exercise and other health-related behaviours may be related despite the lack of evidence elsewhere (Blair, 1988). Somewhat surprisingly, there was no statistical difference in perceptions of the barriers to exercise, although the mean scores showed that slightly greater barriers were perceived by non-exercisers.

The age differences also confirmed many of the findings from Study 1 with older subjects displaying more negative health beliefs. The age $\times$ exercise interaction on exercise intention showed that the greatest intention was by younger exercisers. The commonly held belief that exercise is for the 'young and active' is therefore supported. This could be considered unfortunate since the health benefits of exercise can be achieved at all ages and are likely to be more necessary for older people (Ramlow et al, 1987). Further study is required on the exercise cognitions of older adults.

\section{GENERAL DISCUSSION}

The results suggest that exercisers and non-exercisers clearly differ in their beliefs about health and exercise and that models such as the HBM could be viable starting points for understanding the psychological processes associated with exercise. However, both studies showed that perceptions of vulnerability to ill-health were, paradoxically, associated with sedentary behaviour, at least as far as aerobic exercise was concerned, whereas the HBM predicts that such perceptions are associated with health action. This is likely to be because the HBM is primarily an illness avoidance model rather than a model associated with positive, preventive approach behaviours.

Similarly exercisers were more likely to feel that they were in control of their own health and this has been shown, when combined with high perceptions of value and importance, to be associated with approach behaviours (Wallston et al, 1978; Whitehead and Corbin, 1988). The results in the present study suggest that exercise research should include attributions and variables associated with perceptions of control (King, 1982).

The profiles displayed here may simply be a reflection of the current behaviour patterns of the subjects, rather than being predictive of health and exercise behaviours. However, the results do indicate that beliefs are dissimilar between groups of exercisers and non-exercisers, as well as between gender and age groups. Further research is now required to ascertain how and when such differences are developed in relation to exercise participation and their influence (if any) on physical activity and other health behaviours. Should these results be confirmed, future exercise policy may wish to consider the best ways of modifying the beliefs of non-exercisers. The present results suggest that education programmes are required which specify the safety aspects of exercise, the CHD risk of sedentary lifestyles and motivational strategies which will allow non-exercisers to experience the benefits of activity in a gradual and comfortable way.

Unfortunately, the results presented are based only on cross-sectional retrospective techniques. The shortcomings of this approach are well known. Nevertheless, there is a dearth of research on psychological aspects of exercise and health in Britain, despite our high rates of morbidity and mortality that are associated with certain hypokinetic conditions (e.g. CHD). These exploratory studies, therefore, have attempted to start more sophisticated research into the psychological factors influencing exercise participation.

\section{References}

Bar-On, D and Cristal, N., 1987 "Causal attributions of patients, their spouses and physicians, and the rehabilitation of the patients after their first myocardial infarctions". Journal of Cardiopulmonary Rehabilitation 7: 285-298.

Becker, M. H., Haefner, D. P., Kasl, S. V., Kirscht, J. P., Maiman, L. A. and Rosenstock, I. M., 1977 "Selected psychosocial models and correlates of individual health-related behaviours". Medical Care 15: 27-46.

Biddle, S. J. H., 1986 "The contribution of attrribution theory to exercise behaviour" in J. Watkins, T. Reilly and L. Burwitz (eds.), Sports Science. London: Spon.

Blair, S. N., 1988 "Exercise within a healthy lifestyle" in R. K. Dishman (ed.), Exercise adherence: Its impact on public health. Champaign: Human Kinetics.

British Cardiac Society, 1986. Report of British Cardiac Society working group on coronary disease prevention. London.

Department of Health and Human Services, 1980. Promoting health/ preventing disease: Objectives for the nation. Washington, DC: US Government Printing Office.

Godin, G., Shephard, R. J. and Colantonio, A., 1986 "The cognitive profile of those who intend to exercise but do not". Public Health Reports 101: 521-526.

Janz, N. K. and Becker, M. H., 1984 "The Health Belief Model: A decade later". Health Education Quarterly 11: 1-47.

King, J. B., 1982 "The impact of patients' perceptions of high blood pressure on attendance at screening: An extension of the Health Belief Model". Social Science and Medicine 16: 1079-1091.

Lindsay-Reid, E. and Osborn, R. W., 1980 "Readiness for exercise adoption" Social Science and Medicine 14: 139-146.

Morgan, P. P., Shephard, R. J., Finucane, R., Schimmelfing, L. and Jazmaji, V., 1984 "Health beliefs and exercise habits in an employee fitness programme". Canadian Journal of Applied Sports Sciences 9: 87-93.

Noland, M. P. and Feldman, R. H. L., 1984 "Factors related to the leisure exercise behaviour of 'returning' women college students". Health Education, March/April, 32-36

Noland, M. P. and Feldman, R. H. L., 1985 "An empirical investigation of leisure exercise behaviour in adult women". Health Education, October/ November, 29-34.

O'Connell, J. K., Price, J. H., Roberts, S. M., Jurs, S. G. and McKinley, R., 1985 "Utilising the Health Belief Model to predict dietary and exercising behaviour of obese and nonobese adolescents". Health Education Quarterly 12: 343-351.

Powell, K. E., Spain, K. G., Christenson, G. M. and Mollenkamp, M. P., 1986 "The status of the 1990 objectives for physical fitness and exercise" Public Health Reports 101: 15-21. 
Powell, K. H., Thompson, P. D., Caspersen, C. J. and Kendrick, J. S., 1987 "Physical activity and the incidence of coronary heart disease". Annual Review of Public Health 8: 253-287.

Ramlow, J., Kriska, A. and LaPorte, R., 1987 "Physical activity in the population: The epidemiologic spectrum". Research Quarterly for Exercise and Sport 58: 111-113.

Riddle, P. K., 1980 "Attitudes, beliefs, behavioural intentions and behaviours of women and men toward regular jogging". Research Quarterly for Exercise and Sport 51: 663-674.

Slenker, S. E., Price, J. H., Roberts, S. M. and Jurs, S. G., 1984 "Joggers versus nonexercisers: An analysis of knowledge, attitudes and beliefs about jogging". Research Quarterly for Exercise and Sport 55: 371-378.

Wallston, K. A., Wallston, B. S. and DeVellis, R., 1978 "Development of the multidimensional health locus of control (MHLC) scales". Health Education Monographs 6: 160-170.

Weiner, B., 1986. An attributional theory of motivation and emotion. New York: Springer-Verlag.

Whitehead, J. R. and Corbin, C. B., 1988 "Multidimensional scales for the measurement of locus of control of reinforcements for physical fitness behaviours". Research Quarterly for Exercise and Sport, 59.

\title{
Title:
}

Authors:

\section{PHYSICAL AGENTS FOR PHYSICAL THERAPISTS. 3rd EDITION}

Publisher:

\author{
J. E. Griffin and T. Karsalis \\ Charles C. Thomas, Illinois, 1988 \\ Price: $f \quad 445$ pages Hard cover ISBN 0398053847
}

The preface describes the book as being aimed at physiotherapy students, to be supplemented with lectures and practical work. The range of modalities covered sounds impressive and the authors boast of an up-dated and expanded chapter on nerve and muscle stimulating currents. The first chapter on pain is easy to read and gives a clear description of pain theory, classification and causes. Clear diagrams assist in understanding some of the concepts discussed. Overall it gives a good grounding in pain theory for students as well as revision for qualified physiotherapists. The book then gives an overview of the physical agents at the physiotherapist's disposal i.e. heat, cold, U/S, nerve and muscle stimulating currents, UVL and water. Included is an introduction to the physics of the electromagnetic and accoustic spectron, the most useful section being a comparison of their properties.

Chapter 3 is the up-dated section on nerve and muscle stimulating currents (NMSC). It sets out the physiology of stimulating nerve and muscle with the effects of altering stimulation parameters, e.g. waveform. However, it is not well set out and makes for heavy reading; it would be difficult to quickly check up on facts here. The bulk of the chapter is given over to various clinical applications of NMSC. The author seems to view NMSC as the great panacea for all ills by advocating its use in conditions from upper motor neurone lesions (to prevent muscle atrophy), spinal cord injury to peripheral vascular disease where "the patient will not engage in sufficient activity to make enough use of the calf muscles to keep circulatory insufficiency within tolerable limits". Passive treatment at its height? Disappointingly, no mention is made of recent advances in muscle stimulation e.g. eutrophic stimulation, rather the author still advocates the use of time consuming IDC. There is a good, well referenced section on TENS, describing possible pain relief mechanisms and details of TENS techniques. However, the range of machines described is not readily available in this country. Interestingly, interferential therapy is classed as a form of TENS (high frequency type), but very sparse information on this technique is provided. Finally there is a section covering electrophysiological testing which is well set out and easy to read and a good section on bio-feed-back covering its clinical application e.g. muscle re-education.

Chapter 4 covers the diathermics. It starts with shortwave describing the electrodes (air plate, drum and cable) and the physics and heating mechanism of each. This is a well written section which has important clinical implications as regards choice of electrode for depth of heat and type of tissue heated and would provide a good revision for qualified physiotherapists as well as students. Unfortunately, there is nothing written about pulsed electromagnetic energy which is now widely used. The section on infra-red covers lamps, hot packs and wax and provides a good basic introduction to those techniques. Cold therapy is included here but is very poorly covered in terms of physiological and therapeutic effects.

Ultra-violet light is covered in depth but it is difficult to relate the USA machines to those used in this country. Much of the treatment techniques discussed relate to skin disorders e.g. Psoriasis, with a disappointingly small amount on treatment of wounds. The chapter on ultrasound is disappointing, being based mainly on its use for pain relief by virtue of its heating effect. There is only a short section covering its mechanical effects and use to aid healing. Various treatment techniques are discussed but little emphasis is placed on the possible dangers of ultrasound. I would be dubious about using the stationary technique described because of the danger of standing wave formation and possible stasis of blood flow. The final chapter is on instrumentation and starts with basic laws of electricity which provides a good introduction for students. However the author rapidly gets onto detailed descriptions of individual machine circuits with circuit diagrams which is not necessary for clinical practice.

Overall I found the book hard to read and felt it was not up-to-date with techniques such as Pulsed Electromagnetic Energy, U/S, Interferential: Also many of the techniques are not set in context of being an adjunct to overall patient management which should be made clear, especially for students. I would not advocate buying this book, its useful sections being outweighed by poor, out-dated, difficult to read sections. 